\title{
Interaktions-ergonomische Gestaltung und Kompatibilität von AR-unterstützten Informationsdarstellungen am Beispiel eines Head-Mounted Displays für industrielle Rüstvorgänge
}

\author{
Nils Darwin Abele ${ }^{1} \cdot K_{\text {Karsten Kluth }}^{1}$ \\ Angenommen: 27. September 2021 / Online publiziert: 22. Oktober 2021 \\ ๑) Springer-Verlag GmbH Deutschland, ein Teil von Springer Nature 2021
}

\section{Zusammenfassung}

Im Zuge des technologischen Fortschritts werden zunehmend analoge durch digitale Lösungen ersetzt. In diesem Zusammenhang treten neuartige Visualisierungsmöglichkeiten in den Vordergrund, wie z.B. Augmented Reality (AR). Der Umgang mit AR-basierten Informationsdarstellungen kann jedoch bei inkompatibler Gestaltung zu Performance-Verlusten und erhöhten physischen sowie psychischen Beanspruchungen führen. Bestehende ergonomische und nutzerzentrierte Richtlinien sowie gesetzliche Vorschriften können die Konzipierung und Usability derartiger Systeme aufgrund der rasanten technischen Weiterentwicklung nur bedingt unterstützen. Die vorliegende Ausarbeitung stellt an einem Praxisbeispiel dar, inwieweit eine cyber-physische Rüstapplikation in Form eines Head-Mounted Displays den geltenden interaktions-ergonomischen und kompatibilitäts-bezogenen Standards im Kontext industrieller Tätigkeiten und gesten-gesteuerter, binokularer AR-Systeme entspricht bzw. gerecht wird.

Praktische Relevanz: Die präsentierten Ergebnisse nehmen für die Praxis eine wichtige Rolle ein, da die vorgestellte Systematik Arbeitspersonen bei komplexen Rüst- und Montagevorgängen unterstützen soll. Auf Grundlage dieser Erkenntnisse und mithilfe weiterer Optimierungsmöglichkeiten soll ein prozesssicherer, wertschöpfender und beanspruchungsminimaler Einsatz von AR-Systemen im industriellen Umfeld angestrebt werden.

Schlüsselwörter Augmented Reality · Interaktions-ergonomische Gestaltung · Head-Mounted Display · Industrielle Rüstprozesse · Kompatibilität

\section{Interaction-ergonomic design and compatibility of AR-supported information displays using the example of a head-mounted display for industrial set-up processes}

\begin{abstract}
In the course of technological progress, analog solutions are increasingly replaced by digital ones. In this context, novel visualization options, such as Augmented Reality (AR), are more and more important. However, handling AR-based information displays can lead to performance losses and increased physical as well as psychological strain if the design is incompatible. Due to rapid technological advancements, existing ergonomic and user-centered guidelines and legal regulations can only support the design and usability of such systems to a limited extent. This paper presents a practical example by showing to what extent a cyber-physical set-up application in the form of a head-mounted display complies with or meets the applicable interaction ergonomics and compatibility standards in the context of industrial activities and gesture-controlled, binocular AR systems.
\end{abstract}

Nils Darwin Abele, M.Sc.

Darwin.abele@uni-siegen.de

1 Fachgebiet Arbeitswissenschaft/Ergonomie, Universität

Siegen, Paul-Bonatz-Straße 9-11, 57076 Siegen, Deutschland 
Practical Relevance: The results presented play an important role in practice, since the system presented is intended to support workers in complex set-up and assembly processes. Based on these findings and with the help of further optimization possibilities the aim is to achieve a process-safe, value-adding and strain-minimizing use of AR systems in industrial environment.

Keywords Augmented Reality · Interaction-ergonomic Design · Head-Mounted Display · Industrial Set-up Processes · Compatibility

\section{Technologie-Entwicklung im Produktionsprozess}

Aus der Globalisierung resultierende Absatzmärkte stellen, zusammen mit modernen technischen Möglichkeiten der kostengünstigen Produktion, ein großes Potenzial für industriell gefertigte Produkte dar. Unternehmen müssen ihre Produkte den Marktgegebenheiten und den zunehmend kurzen Produktlebenszyklen anpassen. Mithilfe neuer, fortschrittlicher und qualitativ hochwertiger Produkte zu wettbewerbsfähigen Preisen müssen sie ihre Marktposition erweitern. Neben dem rasanten technischen Fortschritt hat auch die COVID-19-Pandemie gezeigt, dass die Industrie aufgrund sinkender Nachfragen und unterbrochener Lieferketten einen kontinuierlichen Veränderungs- und Anpassungsprozess durchläuft.

Im industriellen Umfeld nimmt demnach, infolge des wachsenden Bedarfs kundenindividueller Produktanforderungen, die Anzahl der Produktvariationen zu. Ein etablierter Versuch diesen Herausforderungen zu begegnen, ist das prozessorientierte Konzept des „Lean Management“ bzw. der „Lean Production“. Es zielt darauf ab, den Anforderungen an eine Flexibilität, mit der Unternehmen auf globale Marktveränderungen reagieren müssen, kundenindividuell gerecht zu werden (Womack et al. 1990). Folgen Unternehmen dem Konzept der „schlanken Fertigung“, sind sie aufgrund des erhöhten Bedarfs an kundenspezifischen Produkten gezwungen, niedrigere Stückzahlen bzw. Losgrößen zu fertigen. Im Vergleich zur Massenproduktion entstehen dadurch höhere Stückpreise. Dieser Umstand führt, insbesondere bei kleinen und mittelständischen Unternehmen (KMU) aufgrund des geringen Standardisierungs- und Automatisierungsgrades, wiederum zu einem Anstieg der häufig noch manuell ausgeführten Rüstvorgänge an Maschinen (Ludwig et al. 2016). Die Komplexität der einzelnen Rüstschritte steigt durch die individuellen Produkte und technischen Anforderungen und beeinflusst somit sowohl den zeitlichen Aufwand als auch die Qualität des Rüstprozesses (Janssen und Möller 2011).

Um erfahrenen, aber insbesondere auch noch unerfahrenen Maschineneinrichtern, auf Grundlage von Theorie und aktuellem Stand der Technik eine umfangreiche Unterstützung gewährleisten zu können, müssen praktische, effiziente und nutzerzentrierte Methoden und Werkzeuge her- angezogen werden. Die Ansätze der Lean Production und der Industrie 4.0 bzw. des Industrial Internet of Things (IIoT) eröffnen im Zuge der digitalen Transformation vielfältige Möglichkeiten der physikalischen Prozessgestaltung, wie z.B. Augmented Reality (AR)-Technologien (Zidek et al. 2020; Birkhan 2008). Das Potenzial zur Verschmelzung nützlicher Informationen mit der realen Umgebung wurde bereits in frühen 1990er-Jahre erkannt (Caudell and Mizell 1992).

Im Zuge der Forschungsbestrebungen haben sich im Wesentlichen zwei Einsatzgebiete herauskristallisiert: Die Kombination konventioneller Eingabemechanismen in Form von Tastatur und Maus mit Displays als Ausgabemechanismus (Gauglitz et al. 2014a) sowie die Verwendung von Touchscreens und HMDs in Verbindung mit Sprachbefehlen (Gauglitz et al. 2014b). Neuere Entwicklungen konzentrieren sich auf die Verwendung von Gesten als mögliche Interaktionsmechanismen für AR-basierte Systeme (Pollalis et al. 2017).

AR-Technologien können in wissensintensiven Umgebungen helfen, einen schnellen, uneingeschränkten und kontextspezifischen Zugang zu Informationen zur Unterstützung von Lernprozessen herzustellen (Klopfer et al. 2005). Insbesondere gilt dieser Sachverhalt für nicht-propositionales Wissen, bspw. in Form verkörperter bzw. nicht verbalisierbarer Handlungen (Gallagher 2010). Erkenntnisse aus der Literatur deuten auch darauf hin, dass AR-Tools die Fähigkeiten und die Wahrnehmung der Nutzer durch eine potenziell nachhaltige Verbesserung der Arbeitsvorgänge, wie z.B. von Montageprozessen (Ong et al. 2008), Wartungsarbeiten (Lee and Akin 2011) oder Schweißarbeiten (Park et al. 2007), positiv unterstützen können.

AR ist somit eine neue Art der Visualisierung, die ortsbezogene Informationen schnell und einfach übertragen kann. Die Forschung zur praktischen Anwendung von AR-basierten Cyber-Physischen Systemen (CPS), z.B. in Form von Datenbrillen oder Head-Mounted Displays (HMD), gilt jedoch bisher als unterrepräsentiert. Gleichzeitig zeigt sie deren Potenzial auf (Bhattacharya and Winer 2019). Aufgrund des relativ frühen Entwicklungsstadiums weisen einige von ihnen z.T. (interaktions-)ergonomische Limitierungen sowohl hinsichtlich der konstruktiven als auch der hardware- und softwareseitigen ApplikationsGestaltung auf, die zwangsläufig die Nutzerakzeptanz be- 
einflussen (Abele und Kluth 2021a, b). Daher ist es von entscheidender Bedeutung, wesentliche Designimplikationen insbesondere für derartige CPS zu definieren.

Aktuelle und frühere Forschungen haben gezeigt, dass neue Technologien, wie insbesondere CPS, in einem sozio-technischen Kontext potenziell zahlreiche unterschiedliche Prozesse unterstützen und erleichtern können. CPS sind Systeme aus eng gekoppelten physischen und digitalen bzw. cyber-physischen Komponenten, die Software, Hardware, Sensoren und Aktoren integrieren. Die allgemeine Definition von CPS impliziert, dass sie verteilte Systeme, die aus physischen und virtuellen Einheiten bestehen, verbinden und verwalten können (Lee et al. 2015). Die Interaktion mit solchen Systemen erfolgt über eine Mensch-Maschine-Schnittstelle, die durch herkömmliche PC-Schnittstellen, Touchscreens oder AR-basierte Technologien realisiert werden kann.

Im industriellen Kontext werden CPS als CPPS (CyberPhysische Produktions-Systeme) bezeichnet. Diese stellen eine Antwort auf die fortschreitende Mechanisierung und Vernetzung von Maschinen und Sensoren dar, die zu großen Datenmengen (Big Data) führen (Kagermann et al. 2013). Diese Systeme werden u. a. für automatische Erkennungs- und Ferndiagnosesysteme (Chen and Tsai 2017), Entwicklungs- und Betriebsphasen in der Produktion (Herterich et al. 2015) sowie für die Lokalisierung von Trends und Möglichkeiten der Optimierung (Shi et al. 2011) eingesetzt. Weitere Forschungen beziehen sich auf die Softwareentwicklung für Smart Factories (Otto et al. 2014) und CPS-basierte Modellierung (Thramboulidis and Christoulakis 2016).

Es bestehen potenzielle Schwierigkeiten und Herausforderungen, die sich aus der Verschmelzung der Interaktion zwischen physischen und virtuellen Welten ergeben, z.B. hinsichtlich der Einbindung von Sensoren, die autark bzw. über eine schlanke Infrastruktur ohne externe Schnittstellen oder vollständig mit menschlichen Schnittstellen erfolgen kann. Diese und weitere Umstände führen zu einer erhöhten interaktionellen und technischen Komplexität. Für neuartige Systeme müssen Prototyping-Tools entwickelt werden, um den Ideenfindungsprozess und das nutzerzentrierte und partizipative Design zu unterstützen (Paelke and Röcker 2015; Monostori 2014). Das gilt auch hinsichtlich der Anwendbarkeit für KMU (Ludwig et al. 2016).

Ein HMD oder eine Datenbrille stellt ein Anzeigegerät dar, d.h. eine lose oder am Kopf befestigte Brille, die Informationen entweder auf einen integrierten, augennahen Bildschirm oder direkt auf die Netzhaut projiziert (Schmauder und Spanner-Ulmer 2014). Mit fortschreitender Entwicklung der Computertechnologie wurde das Zusammenspiel von HMD mit Virtueller Realität (VR) zur interaktiven, intuitiven und verständlichen Darstellung von Informationen in einer geschlossenen, virtuellen Computerwelt verstärkt erforscht (Stanney 2002). Darüber hinaus bedienen sich HMD neben VR der beschriebenen AR-Technologie, die mithilfe des HMD innerhalb der Realumgebung des Menschen virtuelle Elemente bzw. digitalisierte Informationen in das Sichtfeld des Nutzers projiziert. Informationen verbleiben unabhängig von Sitz- bzw. Körperposition im Blickfeld des Nutzers, wodurch eine durchgängige Interaktion - haptisch, auditiv oder gesten-gesteuert - möglich ist.

Die Implementierung derartiger Systeme zielt im betrieblichen Kontext, z. B. in Bezug auf Montage- oder Instandhaltungsvorgänge, u. a. darauf $\mathrm{ab}$, einen permanenten Qualitätsstandard mithilfe einer prozessbegleitenden Verfügbarkeit von Informationen zu ermöglichen (Jensen et al. 2007). Datenbrillen können durch unterschiedliche Anforderungen an Technik, Software, Aufgabengestaltung und letztlich Anwendung auch als kognitive Assistenzsysteme verstanden werden, die wiederum als Hilfesystem zur Bereitstellung von Wissen bzw. Arbeitsinstruktionen, als adaptives Assistenzsystem zur sensorischen Erfassung und Anleitung von Arbeitstätigkeiten sowie als tutorielles Assistenzsystem zur lernförderlichen Arbeitsgestaltung fungieren (Apt et al. 2018).

Einheitliche Definitionen $\mathrm{zu}$ den unterschiedlichen HMD-Typen sind weder in Normen noch in der Fachliteratur zu finden. Aufgrund ihrer Bauart lassen sich HMDs jedoch gemäß den Fach-Informationen (FI) „Nr. 0065“ der Berufsgenossenschaft Holz und Metall (BGHM) in drei Kategorien unterteilen (BGHM-FI Nr. 0065 2019):

1. Binokulare, geschlossene VR-Brillen: Interaktion mit VR-Inhalten isoliert von der realen Umwelt;

2. Monokulare (AR-)Datenbrillen: Visualisierung von augmentierten Informationen auf einem Auge, sodass der Nutzer nur teilweise von der realen Umwelt isoliert ist;

3. Binokulare AR-Brillen: Augmentierte Informationen können zusätzlich zur realen Umwelt mit beiden Augen unter eingeschränkter Isolation wahrgenommen werden.

Neben einer prozessbegleitenden und mobilen Anwendung mit freien Händen, einer sprach- und gesten-basierten Interaktion bzw. Steuerbarkeit sowie der Anbindungsmöglichkeit und Darstellung prozessrelevanter und ggf. nicht einsehbarer Daten sind auch u. U. ein eingeschränktes Blickfeld sowie physische (muskuläre Beanspruchung im Schulter-Nacken-Bereich sowie Komfortbeeinträchtigungen im Kopfbereich) und psychische bzw. kognitive Mehrbeanspruchungen Aspekte, die mit der Nutzung eines HMD einhergehen.

Sämtliche Komponenten müssen in sozio-technischen Systemen der Arbeitswelt aufeinander ausgerichtet werden. Innerhalb eines Mensch-Maschine-Systems sind sensorische und motorische Schnittstellen zur Gewährleistung höchster Sicherheit und optimaler Leistung unter Berück- 
sichtigung menschlicher Eigengesetzlichkeiten hinsichtlich der Informationsaufnahme und -abgabe auszulegen. In einer übergeordneten Ebene bezieht sich dieser Sachverhalt auf eine sinnvolle Zuordnung von Informationen, die auf Anzeigen und Displays analog oder insbesondere im Zuge des fortschreitenden Stands der Technik digital dargestellt werden, zu (meist) motorischen Reaktionen, die an einem Stellteil bzw. an einem Gerät erforderlich werden. Ein hoher Grad an Reiz-Reaktions-Kompatibilität ist dann gegeben, wenn Ursache und Wirkung möglichst einfach miteinander verknüpft sind, d.h. klar erkennbare und eindeutige ReizReaktions-Beziehungen vorliegen.

Im Zuge des Forschungsprojekts „Cyberrüsten 4.0“ wurde am Beispiel von industriellen Rüstvorgängen an Umform- bzw. Biegemaschinen eine prototypische Applikation für eine Mixed-Reality-Technologie unter Verwendung der Microsoft „HoloLens“ entwickelt und evaluiert (Abele et al. 2016; De Carvalho et al. 2018; Hoffmann et al. 2019). Mithilfe dieses HMDs können prozessrelevante Informationen holografisch und kontextspezifisch in das reale Sichtfeld des Nutzers projiziert und mit internen sowie externen Sensordaten und Simulationen angereichert werden.

Industrielles Rüsten umfasst vor dem Start eines Produktionszyklus eine Reihe von vorbereitenden Maßnahmen an einer Maschine oder einem Werkzeug. Es findet zwischen dem Ende der Serienproduktion eines Artikels und dem Beginn der Serienproduktion eines anderen Artikels statt. Aus wirtschaftlicher Sicht ist das ein zentraler und zeitkritischer Prozess: Ohne Rüsten könnte es keine Produktion geben (Kober et al. 2014). Rüstvorgänge an komplexen Produktionsmaschinen, wie z. B. an einer Rotationszugbiegemaschine, stellen einen wissensintensiven Prozess dar, der sich hauptsächlich auf Erfahrungs- und Fachwissen stützt. Derartiges Wissen kann sehr individuell sein und ist sehr schwer zu erfassen und zu teilen (Matthew and Sternberg 2009; Nonaka et al. 2000).

Das Rotationszugbiegen ist ein formgebendes Kaltumformverfahren, das drehende Werkzeugbewegungen ausführt (VDI 3430 2014; Engel und Mathes 2013). Formspeichernde Werkzeuge vollziehen die Formgebung des Halbzeugs, das sowohl als Voll- als auch als Hohlprofil gebogen werden kann. Aufgrund eines umfassenden Werkzeugaufbaus, der i.d.R. sieben wesentliche Werkzeugelemente (Spannfutter, Biegedorn, Gegenhalter, äußere und innere Spannbacke, Biegeform und Faltenglätter) beinhaltet, und der erforderlichen Einstellmaßnahmen unterschiedlicher Maschinenachsen resultiert ein komplexer Rüst- und Einrichtprozess.

\section{Interaktions-ergonomische Gestaltungsanforderungen und Kompatibilität}

Die Sicherstellung eines möglichst uneingeschränkten Material-, Kraft- und Informationsflusses bedarf der wechselseitigen Ausrichtung sämtlicher Komponenten von sozio-technischen Systemen der Arbeitswelt. Die Analyse und Gestaltung der Beziehungen zwischen dem Menschen und einem Arbeitsmittel zielt also auf eine maßliche bzw. anthropometrische Auslegung des Arbeitsplatzes sowie auf die interaktions-ergonomische Konzipierung des Informationsflusses ab. Der technologische Fortschritt bedingt, dass das Einleiten von Informationen in technischen Systemen eine entscheidende Rolle einnimmt. Die an den Menschen zurückgemeldeten Informationen, die auf eine mittelbare oder unmittelbare Informationsausgabe des Arbeitsmittels folgen, werden entsprechend der Aufgabenstellung über unterschiedliche Sinneskanäle verarbeitet. Die übergeordnete Zielsetzung ist auf die Optimierung der Mensch-Arbeitsmittel-Schnittstelle zugunsten eines hohen Wirkungsgrades und einer hohen Zuverlässigkeit ausgerichtet. Dieses Ziel kann wiederum nur bei einem hohen Grad an Kompatibilität erreicht werden (Strasser 1993).

Abb. 1 visualisiert nach Strasser (1993) in einer übergeordneten Ebene die unterschiedlichen Kompatibilitätsarten in einem Mensch-Maschine-System.

Die Reiz-Reiz-Kompatibilität beschreibt die optimale sensorische und motorische Anpassung von technischem Gerät an die menschlichen Eigenschaften in der sensorischen Nahtstelle eines Mensch-Maschine-Systems zur Minimierung des physiologischen Aufwands. Im vorliegenden Fall liegt sie genau dann vor, wenn z. B. natürliche Informationsdarstellungen, d.h. der Blick auf die Maschine, und künstliche Visualisierung durch AR optimal aufeinan-

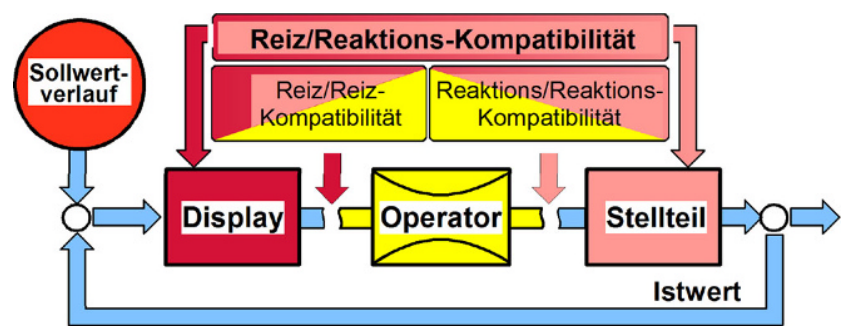

Abb. 1 Reiz-Reiz-, Reaktions-Reaktions- und Reiz-Reaktions-Kompatibilität in einem Mensch-Maschine System bei optimaler sensorischer und motorischer Anpassung von Gerät und menschlichen Eigenschaften sowie sinnfälliger Zuordnung von Ursache und Wirkung bzw. von Anzeige und Stellteileffekt (Quelle: Strasser 1993)

Fig. 1 Compatibility via adaptation of technical equipment to human properties in the sensory and motoric link of a man-machine system (stimulus-stimulus compatibility and response-response compatibility) as well as via corresponding relations between causes and effects, i.e. displays and controls (stimulus-response compatibility) (source: Strasser 1993) 
der abgestimmt sind und sich dadurch die Wirklichkeit und das eingespielte künstliche Abbild weitgehend entsprechen.

\subsection{Menschbezogene Aspekte}

Die über die aktivierten Sinneskanäle der Wahrnehmung (Haptik, Optik, Akustik) aufgenommenen Reize werden in Abhängigkeit von der Stärke, der Motivation und der kognitiven bzw. neurologischen Zustände des Menschen im Sinne der Aufgabenerfüllung bewusst oder unbewusst verarbeitet. Im Anschluss an die Aufnahme und Verarbeitung der Informationen wird die als sinnvoll ausgewählte Reaktion in eine motorische Handlung oder durch Sprache umgesetzt (Bokranz und Landau 1991). Mögliche Fehler im Informationsfluss entstehen, wenn einerseits die Gestaltung eines technischen Hilfsmittels unzureichend erfolgt und andererseits die menschlichen Eigenschaften und Fähigkeiten nicht einem aufgabengerechten Informationsfluss entsprechen (Schmauder und Spanner-Ulmer 2014).

\subsection{Arbeitsmittelbezogene Aspekte}

Der Informationsverarbeitungsprozess eines Arbeitsmittels durchläuft drei Phasen (Schmauder und Spanner-Ulmer 2014): Informationen werden bei ihrer Eingabe durch Betätigung von Stellteilen einer Maschine oder über komplexe Eingabesysteme, die bspw. auch durch eine gestengesteuerte Interaktion mit einer Datenbrille erfolgen kann, übermittelt, anschließend verarbeitet und schließlich über Anzeigen ausgegeben.

Bei Arbeitsmitteln wird zwischen einer Hand- und einer Arbeitsseite unterschieden. Während die Anforderungen der Arbeitsseite, wie z.B. Kraftrichtung, Sicherheit, oder Genauigkeit, die Grundlage für die Gestaltung bilden, ist für eine ergonomische Betrachtung die Handseite als Schnittstelle zum Menschen von entscheidender Bedeutung, z. B. hinsichtlich der feinanalytischen Greifart, Kopplungsart, Handhaltung oder Betätigungsart. Die Interaktion mit AR-basierten HMDs kann berührungslos und gestengesteuert erfolgen. Eine Betrachtung der Kopplungsart sowie der Greifart ist damit obsolet. Die zur Interaktion erforderliche Handhaltung variiert je nach Anforderungen der Datenbrille.

Mithilfe von Anzeigen können Informationen dargestellt werden, die unterscheidbare Sachverhalte angeben (DIN EN 894-2 2008). Ihre Ausprägung hängt davon ab, über welche Sinneskanäle, d.h. optischer, akustischer oder taktiler Sinneskanal, die Informationen zurückgemeldet werden sollen. In Bezug auf AR-unterstützte HMD ist dabei insbesondere die optische Komponente von Bedeutung. Visuelle Informationen, die entweder autark generiert oder über unterschiedliche Funktechniken von einem Computer übertragen werden, werden auf einen augennahen Bildschirm oder direkt auf die Netzhaut projiziert. Die Informationen verbleiben im Sichtfeld des Nutzers, wodurch ein ständiges Interagieren sowie eine Überlagerung von Messgrößen und Programmschritten möglich wird. Je nach Anwendungsszenario und Endgerät erlaubt die technische Infrastruktur funktionale Ergänzungen bzw. Anbindungen an weitere Schnittstellen, z. B. Maschinendaten in Echtzeit.

Zur Gestaltung von Informationen mit einer stark ausgeprägten Reiz-Reiz-Kompatibilität müssen wesentliche hardware- sowie software-ergonomische Gestaltungsregeln beachtet und angewendet werden, um inkompatible Darstellungen und dadurch ausgelöste Fehlbedienungen, Performance-Verluste und physische sowie psychische Beanspruchungen (Cometti et al. 2018) auf ein Minimum zu reduzieren.

\subsubsection{Hardware-ergonomische Gestaltungsprinzipien}

Kompatibilität basiert im Sinne habitueller Stereotypen auf der elementaren Erwartung des Nutzers und auf der Stetigkeit bereits erlernter Merkmale hinsichtlich der Beziehung zwischen Anzeige und Wirklichkeit. Eine kompatible Anzeige liegt vor, wenn der Dekodierungsaufwand bei der Informationsverarbeitung durch eine entsprechende Gestaltung gering ist. Informationen müssen sichtbar bzw. gut ablesbar und ohne optische Ablenkungen dargestellt werden. Die Funktion der Stellteile sollte ebenfalls erkennbar sein. Neben der Betätigbarkeit, d. h. einer intuitiven Funktionssteuerung, sind auch Gestaltgesetze hinsichtlich einer Gruppierung von Informationsgebern möglichst nach Homogenitätsmerkmalen zu beachten. Diese Gesetze umfassen eine funktionelle und räumliche Anordnung nach Ähnlichkeitskriterien, eine Klarheit der Funktionsverbindungen sowie eine gute Gestalt. Die Gestaltungsregel Kodierung beinhaltet die Zuordnung einer eindeutigen Kennzeichnung zu jeder Funktion. Zur Optimierung der visuellen und haptischen Erkenn- und Unterscheidbarkeit der Anzeigen und Stellteile werden Kodierungen herangezogen, wie z. B. Farben, Formen, Größen, Positionen oder Piktogramme bzw. Symbole. Um den beabsichtigten Nutzen einer sicheren und unmittelbaren Identifizierung von Kodierungen sicherzustellen, sollten diese einheitlich verwendet werden. Eine farbliche Kodierung wird häufig über die Verwendung von Ampelfarben (grün: Sicherheit; gelb: Vorsicht bzw. Achtung; rot: Gefahr) generiert. Während Formen intuitives Handeln ermöglichen können, kann ein subjektives Rangverständnis der Größe von Bedienteilen unterschiedlicher Hersteller die Weitergabe kodierter Informationen (negativ) beeinflussen. Mithilfe von Kodierungen können außerdem Funktion, Zustand oder Wirkung von Stellteilen kodiert werden. Eine Funktionskodierung impliziert die mit dem Stellteil zu bedienende Funktion. Der Zustand einer Funktion wird über eine geeignete Anzeige übertragen. Bei 
der Wirkungskodierung wird der Nutzer über die Auswirkungen der Bestätigung eines Stellteils informiert.

\subsubsection{Software-ergonomische Gestaltungsprinzipien}

Ziel der Software-Ergonomie ist die Anpassung der Software bzw. der User-Interfaces an physische und psychische Eigenschaften als auch an Kenntnisse und Gewohnheiten des Nutzers (DGUV Information 215-450 2021). Die Darstellung von Bild und Text soll hierbei den Bedürfnissen des Nutzers angepasst sein, um eine beanspruchungsminimale und effiziente Interaktion zu ermöglichen (Heeg 1988; Hacker 1987). Bei der Gestaltung der Software soll also ein hohes Maß an Usability, d.h. Benutzerfreundlichkeit und einfacher Bedienbarkeit, gewährleistet sein. Eine Vielzahl an technisch realisierbaren Programmkapazitäten ist zwar möglich, aber nicht immer sinnvoll. Ein „Information Overload" ist in jedem Fall zu vermeiden.

Ärger, Frustration, Fehler und Zeitverlust sind neben physiologischen Kosten, wie Kopfschmerzen oder vorzeitiger Ermüdung, Folgen einer nicht ergonomisch gestalteten Software (Navas 2015). Das menschliche Verarbeitungsvermögen von Informationen und Farben sowie das Kurzzeit- und Arbeitszeit-Gedächtnis nehmen bei der Gestaltung der Software eine sehr wichtige Rolle ein. Die Wahrnehmung und das Auffinden von Informationen sind genauso zu berücksichtigen wie das Verständnis von Begriffen und Kürzeln, die Beibehaltung von Icons und Symbolen, die Erwartung von bestimmten Funktionalitäten sowie die Gewöhnung an die Anwendung (Balzert 1987). Das Normenwerk DIN EN ISO 9241-110 (2020) sowie die Information ,215-450“ der Deutschen Gesetzlichen Unfallversicherung (DGUV) bauen auf diesen Aspekten auf und geben für die Ergonomie der Mensch-System-Interaktion wesentliche, nachfolgend aufgeführte Grundsätze zur Dialoggestaltung vor, um den Software-Entwicklern und -Anwendern eine möglichst benutzerfreundliche und gebrauchstaugliche Konzeption der Interfaces ermöglichen zu können:

- Aufgabenangemessenheit: Unterstützung des Nutzers zur effektiven und effizienten Erledigung seiner Arbeitsaufgabe.

- Selbstbeschreibungsfähigkeit: Angebot angemessener Informationen zur Offenlegung der Systemfähigkeiten und Nutzungsmöglichkeiten.

- Erwartungskonformität: Erwartungskonformer Dialog durch vorhersehbares Nutzerverlangen des Nutzungskontextes sowie durch allgemein anerkannte Konventionen.

- Lernförderlichkeit bzw. Erlernbarkeit: Unterstützung bei der Entdeckung von Fähigkeiten und deren Verwendung, Explorieren (,Ausprobieren“) des interaktiven Systems und Lernunterstützung.
- Steuerbarkeit: Kontrolle über Benutzungsschnittstelle sowie die Interaktion inklusive der Geschwindigkeit, Abfolge und Individualisierung der Benutzer-SystemInteraktion.

- Robustheit gegen Benutzungsfehler: Visualisierung von Fehlermeldungen und Anleitungen zur Fehlerbehebung, sodass eine unmittelbare Fehlerkorrektur erfolgen kann.

- Benutzerbindung: Darstellung von Funktionen und Informationen auf einladende und motivierende Weise zugunsten einer kontinuierlichen Systeminteraktion.

DIN EN ISO 9241-112 (2017) führt ergänzende Grundsätze auf, die allgemeine Gebrauchstauglichkeitsprobleme behandeln, welche während der Entwicklung von Benutzungsschnittstellen bei der Informationsdarstellung entstehen. Um den Usability-Anforderungen, d.h. Anforderungen hinsichtlich der Gebrauchstauglichkeit und Benutzerfreundlichkeit von Produkten bzw. Anwendungen, gerecht zu werden, sind dabei die Zielkriterien „Effektivität“, ,Effizienz“ und „Zufriedenstellung“ zu erfüllen. Zu den genannten Grundsätzen zählen Entdeckbarkeit, Ablenkungsfreiheit, Unterscheidbarkeit, eindeutige Interpretierbarkeit, Kompaktheit und Konsistenz.

\section{Designprozess eines AR-basierten CPS}

Der als Anwendungsfall dienende Rüstprozess an einer Rotationszugbiegemaschine war auf die Gestaltung eines AR-basierten CPS ausgerichtet, das Maschineneinrichtern dazu verhelfen kann, Wissen und Fachkenntnisse über kontextspezifische Rüstprozesse effizient, leistungssteigernd und möglichst beanspruchungsminimal zu teilen und gleichermaßen zu beziehen. Dazu wurde eine Designfallstudie (Wulf et al. 2015) durchgeführt. Sie stellt ein methodisches Konzept zur praxisorientierten Erforschung bzw. Gestaltung innovativer technischer Systeme bzw. Designimplikationen, sogenannter Artefakte, dar. Aufgrund möglicher, teils stark unterschiedlicher Vorgehensweisen des Menschen im Zuge praktischer Tätigkeiten sollte das Design eines Informations- und Kommunikationstechnik (IKT)Tools auf den Menschen ausgerichtet sein. Dabei wird ein nutzerzentrierter Design-Ansatz verfolgt, der die Wechselwirkungen sozialer Praktiken mit den Artefakten analysiert. Neben der empirischen Analyse des Handlungsfeldes erfolgt die Entwicklung einer innovativen Darstellungsform und die Evaluierung in der Praxis.

\subsection{Designimplikationen}

Die Auswertung der empirischen Studie hat gezeigt, dass jede Lösung aufgrund des Mangels an „Expertenwissen“ 
und der fehlerintoleranten Produktionsumgebung relevante Informationen zeitnah und mit minimalen Fehlern bereitstellen sollte, um letztlich die Produktionskosten niedrig zu halten. Das prozessrelevante Datenmaterial muss außerdem angemessen visualisiert werden. Sowohl die schrittweise Erfassung als auch die Wiedergabe der Instruktionen bzw. des Lehrmaterials in Form von Text, auditiven Kommentaren sowie bild- und videobasierten Inhalten sollte aus der Ich-Perspektive, z. B. mit Hilfe von HMDs, realisiert werden, um den Einsatz beider Hände für die arbeitenden Personen zu gewährleisten. Darüber hinaus trägt eine geeignete Sensorik durch die Überwachung des Arbeitsbereichs dazu bei, die Unsicherheit der Benutzer zu verringern. Damit erhöht sich die Effizienz der Instruktion durch Fehlervermeidung und Stressreduzierung. Die schrittweise Erfassung der Rüstschritte verhilft in Kombination mit der Aufzeichnung von Wegstrecken außerdem zu rollierenden möglichen Optimierungsmaßnahmen des gesamten Rüstvorgangs. Zusammenfassend wurden sechs Themen als wesentliche Designimplikationen identifiziert (vgl. Abb. 2): Verwendung von Sensoren zur Erfassung von Realdaten, Verwendung von digitalen Simulationen in Situationen, in denen keine realen Daten zur Verfügung stehen, Unterstützung der Aggregation digitaler Daten zu Arbeitspraktiken, Unterstützung des Austauschs von Wissen und Expertise, Unterstützung der Datenkonfiguration und Bereitstellung von praktikabler Interaktion in industriellen Kontexten. Diese Themen basieren nicht nur auf der Datenanalyse der DesignWorkshop-Daten, sondern auch auf den Daten der empirischen Analyse. In der nachfolgenden Abbildung werden themenbezogene Interfaces aus Gründen der Darstellbarkeit nur exemplarisch aufgezeigt.

Interessanterweise stimmen die identifizierten Themen weitgehend mit der 5-Ebenen-Architektur für das Design

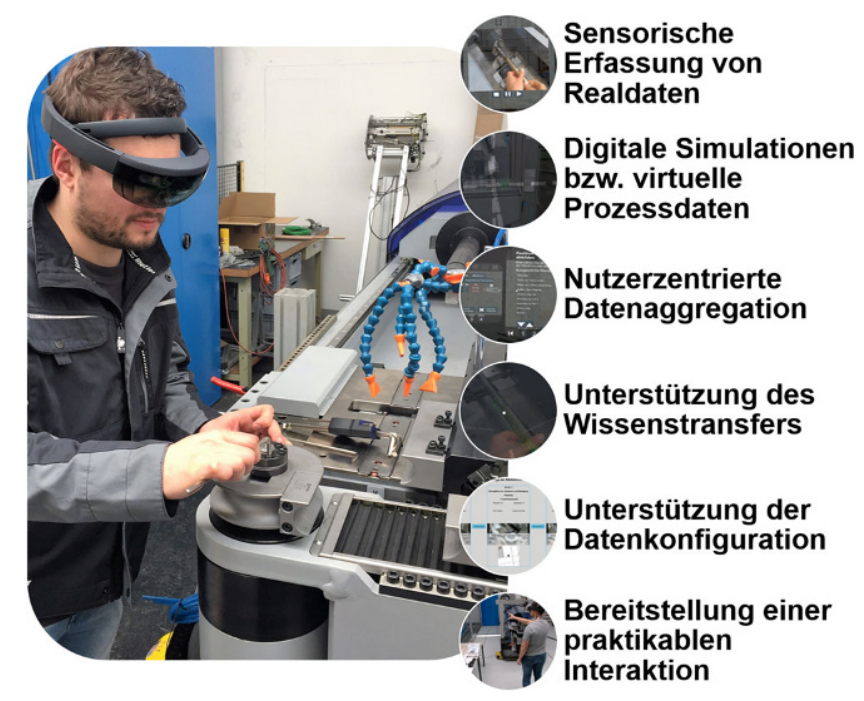

Abb. 2 Designimplikationen für ein AR-basiertes CPS-Rüsttool Fig. 2 Design implications for an AR-based CPS set-up tool von CPS von Lee et al. (2015) überein. Lee et al. (2015) stellen eine Struktur und Architektur für CPS vor, die mit der Datenerfassung beginnt, über eine Analyse hinausgeht und mit einer wertschöpfenden Wissenskonfiguration endet. Diese Architektur soll als Leitfaden für die Implementierung von CPS in der Industrie dienen, die wiederum durch praktische Anwendungen und Techniken ergänzt werden soll, welche die theoretische Architektur mit praktischen Umsetzungsmöglichkeiten untermauern. Nichtsdestotrotz erweitern die vorliegenden Ergebnisse diese Architektur, da sie über die Betrachtung der technischen Aspekte hinausgeht und sich mit den sozio-technischen Aspekten des Designs und den Möglichkeiten der Schaffung einer Umgebung, in der Wissenstransferprozesse stattfinden können, auseinandersetzt. Unabhängig davon galt es im Zuge der Interface-Gestaltung, den geltenden Normen, Richtlinien, Vorschriften sowie Standards der Interaktions-Ergonomie gerecht zu werden, um einen möglichst hohen Grad an Kompatibilität zu erreichen. Die zentralen ergonomischen Gestaltungsaspekte des CPS sollten sich daher daran orientieren, dass die Interaktion des Maschinenbedieners während des gesamten Prozesses nicht negativ beeinflusst wird. Dabei geht es nicht um eine möglichst einfache Interaktion, sondern um eine sinnvolle Anpassung der Komplexität an den Einsatzbereich (Janlert und Stolterman 2010).

\subsection{Interface-Gestaltung und Workflow}

Die vorgestellten Designimplikationen wurden in Form einer prototypischen Rüst-Applikation bzw. -Software namens ,Expert to Go“ für die binokulare, sprach- und gestengesteuerte AR-Datenbrille „Microsoft HoloLens“ der ersten Generation umgesetzt. Die Microsoft HoloLens ist eine binokulare Windows-10-betriebene Mixed-Reality-Brille (N.N. 2019). Die Grundfunktionen des Tools lassen sich in einen Aufnahme- (,Writing Mode“) und einen Wiedergabemodus (,Reading Mode“) unterteilen. Das „Schreiben“ ermöglicht dem Experten die Aufzeichnung prozessrelevanter Inhalte. Mithilfe des ,Lesen“-Modus werden die Rüstanweisungen kontextspezifisch und schrittweise visualisiert sowie mit internen und externen Sensordaten und Simulationen angereichert.

In Bezug auf das vorliegende Praxisbeispiel nimmt insbesondere die Gestaltung der Interfaces bzw. der Hologramme, die durch sensorische Eingangswerte und deren Visualisierung angereichert bzw. unterstützt wird, als elementare Darstellungsform der HoloLens einen wichtigen Stellenwert ein. Das nachfolgende Abb. 3 visualisiert entsprechende Basis-Hologramme.

Aus Gründen der Darstellbarkeit wird vornehmlich Bezug auf den „Lesen“-Modus genommen. Der Workflow des sogenannten „Static Expert Module“ beginnt mit der Anmeldung des Nutzers und der Auswahl der ab- und aufzu- 

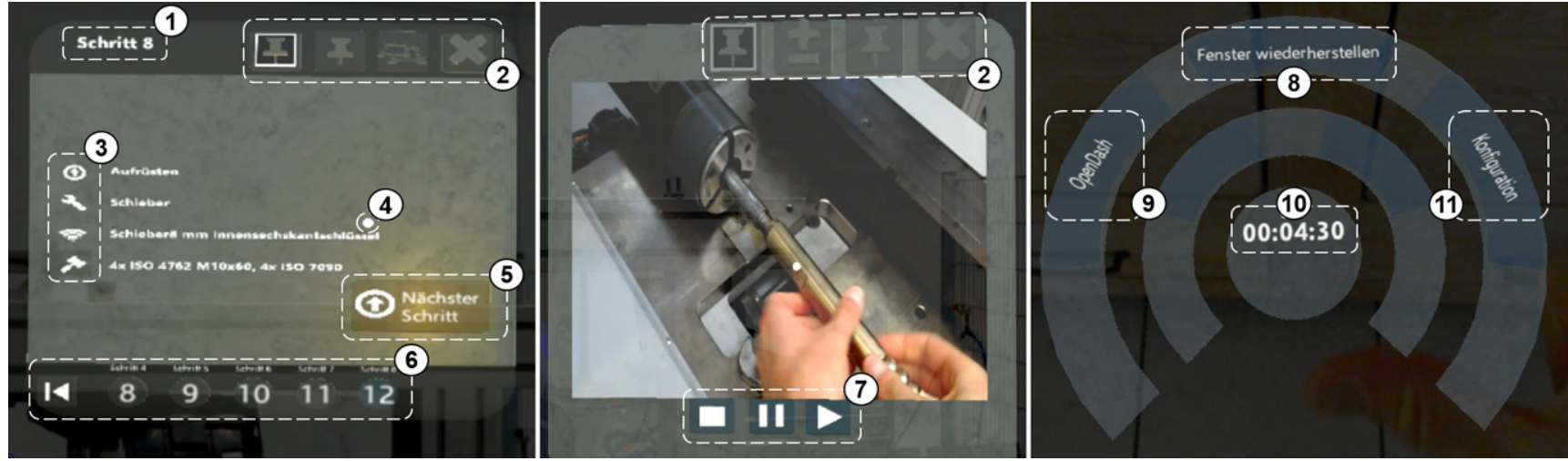

Abb. 3 Basis-Hologramme des „Expert to Go“: „Parent“- (links) und „Child“-Hologramm (mittig) sowie „Home“-Menü (rechts)

Fig. 3 Basic holograms of the "Expert to Go": "Parent" (left) and "Child" hologram (center) as well as "Home" menu (right)

rüstenden Artikel. Im Nachgang wird der Anwender mithilfe der Hologramme Schritt für Schritt durch die Rüstinstruktion geleitet. Dabei kann er den aktuellen Prozessstatus über eine entsprechende Anzeige (1) sowie über eine sogenannte „Progress bar“ (6) einsehen. Während ein Interaktions-Button zum nächsten Rüstschritt überleitet (5), verhilft die Progress bar dazu, vorherige Rüstschritte bzw. Interfaces einzusehen. Die Hologramme beinhalten außerdem sogenannte „Window Commands“ (2), um die Interfaces hinsichtlich Größe und Position manipulieren zu können. Über das „Parent“-Hologramm erhält der Nutzer textliche Informationen (3) zum Arbeitsprozess, d.h. zum Rüstvorgang, Werkzeug, Montagehilfsmittel und zu Befestigungselementen. Er hat darüber hinaus die Möglichkeit, „Child“Hologramme einzusehen. Maximal drei Fenster können zusätzlich zum Parent-Hologramm geöffnet werden. Diese enthalten bild- und videobasierte Inhalte mit zugehörigen Steuerelementen (7). Darüber hinaus kann noch ein weiteres Interface zur Visualisierung von z.B. Simulationen, Zeichnungen, Explosions- oder 3D-Ansichten des zu fertigenden Artikels eingesehen werden. Über ein sich vor den Füßen des Nutzers befindliches „Home“-Menü kann der Nutzer geschlossene Fenster wiederherstellen (8), Maschinendaten (9) sowie die Rüstzeit einsehen (10) und Darstellungsformen konfigurieren (11). Das CPS wird zusätzlich durch virtuelle und interaktive 3D-CAD-Hologramme der Werkzeuge bzw. der Maschine ergänzt, die sich mit den realen Elementen überlagern.

Nach Beendigung des mechanischen Rüstprozesses kontrolliert eine 3D-Kamera die korrekte Montagepositionen der Werkzeuge, sodass der Nutzer auf Grundlage eines visuellen Soll-Ist-Vergleichs etwaige Änderungen vornehmen kann.

Die Interaktion mit der HoloLens erfolgt gesten- oder sprachgesteuert - sie kann jedoch auch über ein kabelloses Eingabegerät, den sogenannten „Clicker“, durchgeführt werden. Durch die im Rahmen des Rüstvorgangs bestehen- de Erfordernis des freihändigen Arbeitens und aufgrund der in Industrieumgebung u.U. aufkommenden hohen Lärmexpositionswerte wurde innerhalb des Forschungsprojektes nur eine gesten-gesteuerte Interaktion betrachtet. In dem $120^{\circ}$-Sichtfeld der HoloLens können zwei wesentliche Gesten-Steuerungen erkannt werden: der „Air Tap“ und die „Bloom“-Geste. Bei der „Air Tap“-Ausführung wird zunächst ein Arm cranial-ventral auf Gesichtshöhe ausgelenkt. Anschließend soll sich der erhobene Zeigefinger wie bei einem „Mausklick“ in caudale Richtung bewegen, um dann wieder in die Ausgangsposition zurückzukehren. Die HoloLens erkennt die Blickrichtung des Nutzers mithilfe des sogenannten „Gazes“ oder „Gaze Point“ (4). Der fixe Punkt, der sich in der Mitte des Sichtfelds befindet und nur mit Kopfbewegungen (nicht via Eye-Tracking) gesteuert werden kann, ist entsprechend mit der Funktionsweise eines Maus-Cursors vergleichbar. Ein Objekt kann ausgewählt bzw. angeklickt (,Short Tap“) oder, vergleichbar mit einer gedrückten linken Maustaste, gezogen werden („Long Tap“). Durch die „Bloom“-Geste kann der Nutzer darüber hinaus das Start- bzw. Hauptmenü öffnen. Dafür wird die Hand zunächst zu einer Faust geballt und anschließend geöffnet.

\section{Ergonomische Gestaltungsparameter und Kompatibilität von AR-unterstützten Informationsdarstellungen am Beispiel einer prototypischen Rüstapplikation}

Anhand ausgewählter, exemplarischer und nachfolgend aufgeführter Beispiele soll verdeutlicht werden, dass die wesentlichen Grundsätze zur interaktions-ergonomischen Gestaltung von informatorischen Arbeitsmitteln unter besonderer Berücksichtigung der Kompatibilität eines MenschMaschine-Systems bestmöglich verfolgt wurden. 
Für eine benutzerfreundliche, gebrauchstaugliche und letztlich ergonomische Gestaltung einer Mensch-SystemInteraktion sollten insbesondere die beschriebenen internationalen Richtlinien der DIN EN ISO 9241-Norm zur Mensch-Computer- bzw. Mensch-System-Interaktion sowie das gesetzliche Regelwerk um die Verordnung über Arbeitsstätten (ArbStättV, 2004) und die DGUV-Informationen zu Bildschirmarbeitsplätzen (DGUV Information 215-410 2019) und zur Software-Ergonomie (DGUV Information 215-450 2021) beachtet werden. Im Folgenden werden am Beispiel der Rüst-Applikation insbesondere die übergeordneten Empfehlungen zur Dialoggestaltung bzw. zu Interaktionsprinzipien (DIN EN ISO 9241-110 2020) sowie die allgemeinen Grundsätze zur Informationsdarstellung (DIN EN ISO 9241-112 2017) in Gänze betrachtet. Aus kapazitiven Gründen wird aber nur vereinzelt auf spezielle thematische Schwerpunkte inklusive etwaiger normativer Bezüge verwiesen.

\subsection{Hardware-ergonomische informatorische Gestaltung}

Die Sichtbarkeit gilt als wichtigstes Gestaltungsziel für eine hardware-ergonomische informatorische Gestaltung. Dementsprechend waren die Hologramme der HoloLens so anzuordnen, dass sie die prozessrelevanten Informationen gut lesbar und eindeutig darstellen. Zunächst können durch das in dem HMD eingebaute „Liquid Crystal Display“ (LCD, DIN EN ISO 9241-303 2011) Informationen reflexionsarm, flimmer- und weitgehend strahlungsfrei eingesehen werden. Ein helles und kontrastreiches Bild, das eine gute Lesbarkeit sicherstellt sowie ein frühzeitiges Ermüden der Augen vermeidet, kann allerdings durch starken Lichteinfall (über 2000 lx) beeinträchtigt werden. Das Gütesiegel „TCO Certified Displays“, das die Bildschirmqualität unter Berücksichtigung ergonomischer Richtlinien bewertet, fordert für Anzeigeleuchtdichten mindestens $150 \mathrm{~cd} / \mathrm{m}^{2}$. Ein entsprechendes Qualitätssiegel trägt die HoloLens der ers- ten Generation zwar nicht, übersteigt mit $320 \mathrm{~cd} / \mathrm{m}^{2}$ jedoch den Referenzwert deutlich.

Die zentrierte Darstellung bzw. Anordnung der Hologramme vereint die wesentlichen Informationen in unmittelbarer Nähe zueinander. Das AR-Prinzip der HoloLens erlaubt eine individualisierbare Platzierung der Fenster (DIN EN ISO 9241-125 2017). Die Hologramme können entweder an ebene Flächen im Umfeld des Nutzers fixiert oder horizontal im Blickfeld des Anwenders platziert werden. Der standardgemäß eingestellte ,Tag along“-Modus folgt dem Sichtfeld bzw. dem sogenannten „Field of view“ des Nutzers (HoloLens 1: $34^{\circ}$ ). Die 1. Generation der HoloLens weist mit $1280 \times 720$ Pixel (pro Auge) und durch ihre binokulare Visualisierung eine große Bildschirmauflösung auf und liegt damit über dem ergonomischen Richtwert von $1600 \times 1200$ Pixel für ein 21-Zoll-Display (DGUV Information 215-410 2019). Durch eine automatische PupillenEntfernungs-Kalibrierung werden die holografischen Informationen bei entspannter Mittellage der Augen ca. $15^{\circ}$ unterhalb der horizontalen Blickachse visualisiert. Durch den Tag-along-Modus ist das Sichtfeld der HoloLens der Sehrichtungsklasse III (geeignete Sicht auf LCD-Display bei beweglicher Kopfposition) zuzuordnen.

Mit der Betätigbarkeit wird eine intuitive Ausführung der Funktionen angestrebt. Der Nutzer interagiert mit der HoloLens im vorliegenden Praxisbeispiel gesten-gesteuert. Diese (neuartige) Interaktionsweise bedingt, dass nicht zwingend von einer intuitiven Bedienung ausgegangen werden kann, sondern vielmehr eine Einweisung sowie ein Gewöhnungsprozess erfolgen muss. Durch die Einblendung von 3D-Hologrammen der Maschine bzw. Werkzeuge werden außerdem reale (zu rüstende) Elemente durch virtuelle (CAD-)Daten ergänzt, um dem Nutzer eine unmissverständliche und unmittelbare Funktionsdurchführung zu gewährleisten (vgl. Abb. 4).

Diese Gestaltungsmaßnahme unterstützt zudem das Prinzip der räumlichen Kompatibilität. Durch diese Visualisierungsart, die durch eine dynamische Anzeige der Mon-

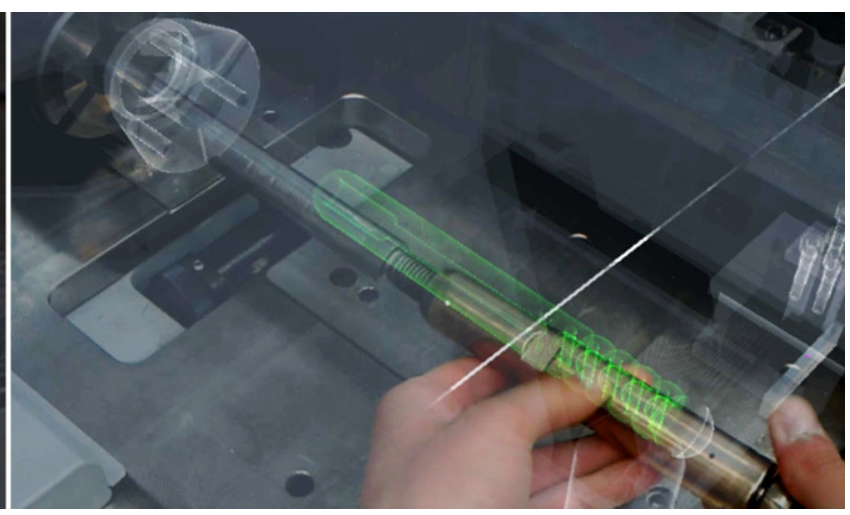

Abb. 4 Wiedergabe der erstellten Rüstdaten in Form der interaktiven 3D-Hologramme der Werkzeuge

Fig. 4 Playback of the created set-up data in the form of the tools' interactive 3D holograms 
tagebewegungsrichtung der einzelnen Werkzeuge ergänzt wird, werden außerdem bewegungskompatible Merkmale gewährleistet. In den Interfaces der Rüst-Applikation werden multimediale bzw. text-, bild- und video-basierte Inhalte zum jeweiligen Rüstschritt dargestellt. Durch diese visuelle Darbietung der prozessrelevanten Informationen, die räumlich-analoge Verarbeitung der Informationen sowie die geforderte und durch den Montageprozess realisierte manuelle Reaktion konnte außerdem eine modalitätsbezogene Kompatibilität generiert werden.

Der Mensch kann zu bestimmten Gegebenheiten verschiedene Teilreize miteinander verbinden und diese im besten Fall nach Homogenitätsmerkmalen gruppieren. Dieser Vorgang trägt zu einer effizienteren Informationsübermittlung an den Nutzer bei. Durch die Platzierung der einzelnen Interfaces in unmittelbarer Nähe zueinander und zum Montageort werden diese als zusammengehörige Gruppe wahrgenommen. Die in ihrer Struktur identische Gestaltung der Basis-Hologramme (vgl. Abb. 4) verfolgt außerdem das Prinzip der Ähnlichkeit. Durch klare Konturen und Trennlinien werden außerdem die Gesetze der guten Gestalt sowie der Geschlossenheit beachtet. Der Nutzer wird damit Schritt für Schritt durch die Arbeitsaufgabe geführt, sodass außerdem das Gesetz der Kontinuität bzw. Fortsetzung implementiert wurde. Das Prinzip der gemeinsamen Bewegung wird dadurch beachtet, dass die Hologramme dem Blickfeld des Nutzers folgen.

Ferner soll die Kodierung zur Verbesserung einer visuellen und taktilen Erkenn- und Unterscheidbarkeit der Anzeigen und Stellteile beitragen. Darüber hinaus sollten Informationen derart dargestellt werden, dass eigenständige Elemente bzw. Gruppen eindeutig voneinander zu unterscheiden sind. In Bezug auf das vorliegende Beispiel wird keine haptische Interaktion, sondern vielmehr eine gesten-gesteuerte Interaktion berücksichtigt. Das „klassische“ analoge Stellteil wird in diesem Fall also durch ein digitales Eingabegerät bzw. ein interaktives Steuer- oder Bedienelement in einer grafischen Benutzeroberfläche (Graphical User Interface, GUI) mittels allgemein bekannter Symbole oder Piktogramme ersetzt. Somit können auch weniger technikaffine Personen oder solche, die der Systemsprache nur unzureichend mächtig sind, prozesssicher mit der Applikation interagieren.

Bestimmte Symbole bzw. Piktogramme sind durch ihren Bekanntheitsgrad unmissverständlich (vgl. Abb. 5).

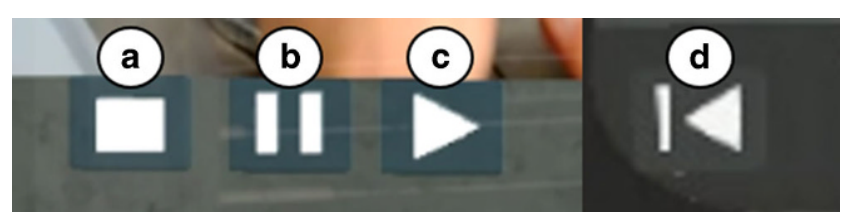

Abb. 5 Unmissverständliche Piktogramme bzw. Symbole

Fig. 5 Unambiguous pictograms or symbols
Dazu zählen u.a. Symbole für das Stoppen (a), Pausieren (b) und Abspielen (c) des jeweiligen Rüst- bzw. Montagevideos oder ein in Linksrichtung gesetzter Pfeil (d), der den Nutzer zum zuvor eingesehenen Fenster bzw. zum vorherigen Rüstschritt führt.

Bei der Kodierung mittels Farbe wurde zunächst darauf geachtet, dass die Interfaces als reiz-reiz-kompatible sensorische Nahtstelle in einem Mensch-Maschine-System möglichst eine Minimierung des Umadaptionsaufwands des Auges beim Blickwechsel von den holografischen Inhalten auf die Realumgebung bzw. die Maschine und Werkzeuge garantieren können. Dazu wurde den Anforderungen an elektronische optische Anzeigen entsprechend eine in Grautönen gehaltene Negativdarstellung (DIN EN ISO 9241303 2011) sowie große und helle bzw. weiße Beschriftungen (DIN EN ISO 9241-112 2017) gewählt, die in Kombination eine ausreichend kontrastreiche Darstellung und Unterscheidbarkeit der holografischen Inhalte bietet. Während sich Positivdarstellungen für hell adaptierte Augen eignen, sind dunkel adaptierte Augen der Negativdarstellung zuträglich. Da die Hologramme mit einem additiven Lichtsystem erstellt werden, ist heller bzw. weißer Text gut lesbar. Somit kann der Nutzer die Inhalte besser fokussieren und wird weniger durch Umgebungseinflüsse abgelenkt. Darüber hinaus wurde eine angemessene und einheitliche Auswahl von Farben, wie z. B. grau, grün und blau, berücksichtigt. Damit soll einerseits eine beruhigende psychische Stimmungslage erzeugt werden (Bullinger 1994) und andererseits eine Gleichmäßigkeit der Leuchtdichte, des Kontrastes und der Farbe in Abhängigkeit der sich ständig ändernden Sehrichtung gewährleistet werden (DIN EN ISO 9241-303 2011). Für die Erstellung der Rüstinstruktionen sind für die einzelnen Maschinen-, Werkzeug-, Programmierungs- und Prüfvorrichtungskomponenten multimediale Inhalte vorzusehen. Beispielsweise müssen für Werkzeugkomponenten Montagerichtungen in horizontaler oder vertikaler Richtung definiert werden (vgl. Abb. 6). Das ausgewählte Werkzeugelement wird dabei grün, die Achsrichtungen grau und die angewählte Achse in einem helleren Blauton visualisiert. Während der Nutzung des ,Lesen“-Modus, d.h. während des laufenden Rüstvorgangs, wird dann das aktuell zu rüstende Werkzeug in der Farbe „Grün“ hervorgehoben, während die übrigen Werkzeuge in „Weiß“ gekennzeichnet sind (vgl. Abb. 4). Die Deckkraft der Hervorhebungen ist gering gehalten, um Blendung zu vermeiden.

Sobald eine Interaktionsschaltfläche, wie bspw. das Abspiel-Widget des Videos, mit dem Gaze Point angewählt wird, hebt sich das Icon farblich (in einem leichten Gelbton) von den blau-grauen Symbolen ab. Als nachteilig ist in diesem Zusammenhang jedoch zu erwähnen, dass sich der weiße Gaze Point insbesondere mit dem Video-Steuerelement, das ebenfalls weiß dargestellt wird, kontrastlos überlagert (vgl. Abb. 7). 
Abb. 6 Anlegen von Rüstinstruktionen; hier: Festlegung der horizontalen Montagerichtung eines Werkzeugs

Fig. 6 Creation of set-up instructions; here: Determination of the tool's horizontal mounting direction

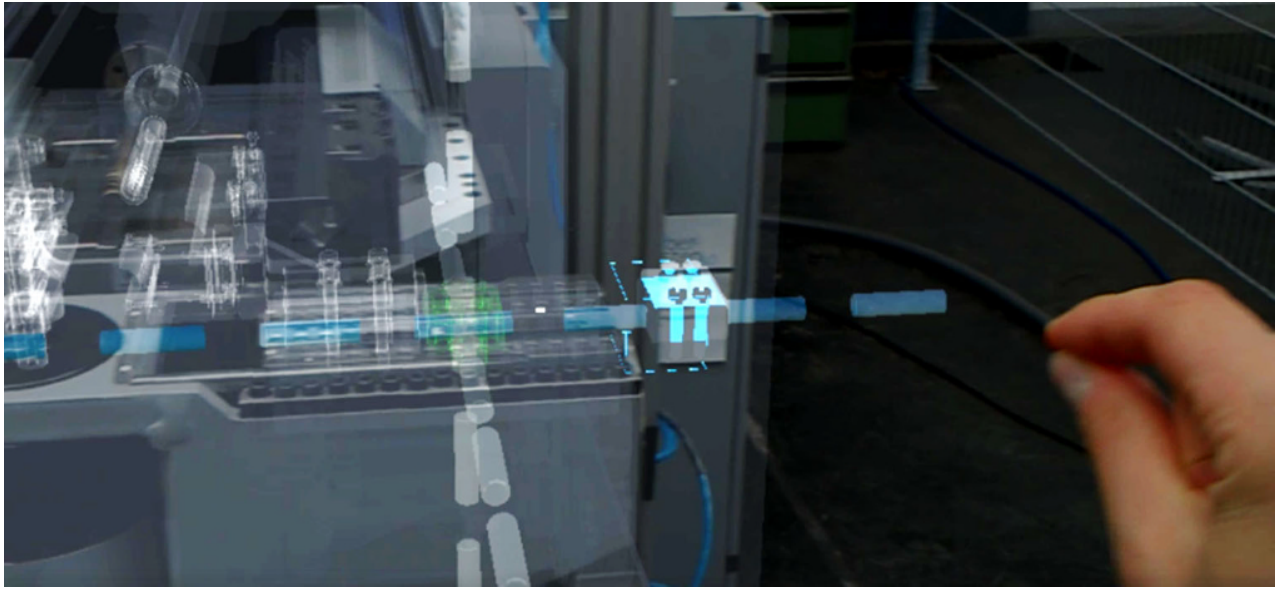

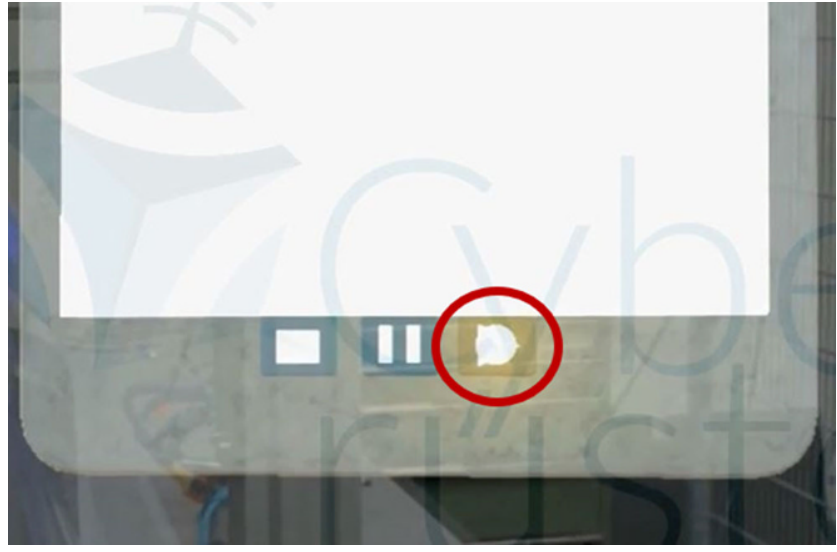

Abb. 7 Kontrastlose Überlagerung des Gaze Points mit einer Interaktionsschaltfläche

Fig. 7 Non-contrast overlay of the Gaze Point with an interaction button

Nach DIN EN ISO 9241-303 (2011) erfüllt die gewählte serifenfreie Schriftart „Arial“" weiterhin ergonomische Anforderungen hinsichtlich des Zeichenabstands sowie der Nutzerakzeptanz für elektronische optische Anzeigen. Für HMDs existieren hinsichtlich der Schriftgröße bisher weder in Teil 303 (2011) und Teil 307 (2008) der DIN EN ISO 9241 noch in der DGUV-Information 215-410 (2019) entsprechende Vorgaben. Das Hologramm der HoloLens erscheint dem Nutzer in $45 \mathrm{~cm}$ Abstand zur Linse. Im Vergleich zu Richtlinien, die für Bildschirme gelten, konnte mit einer Zeichenhöhe von 5,5 mm und einem Schriftgrad von 15 Punkten für das vorliegende Szenario eine bequem lesbare Zeichengröße geschaffen werden.

\subsection{Software-ergonomische informatorische Gestaltung}

Die verwendete Applikationssoftware „Expert to Go“ ist u. a. aufgaben-angemessen, da sie den Nutzer anhand der Basis-Hologramme schrittweise durch den Rüstprozess lei- tet. Die multimedialen und kontextspezifischen Informationen zur Nutzer-System-Interaktion basieren auf den charakteristischen Eigenschaften der (Rüst-)Aufgabe und liefern eine kompakte Informations- und Aktionsdichte. Über das Home-Menü kann der Maschineneinrichter jederzeit die bisher benötigte Rüstzeit einsehen. Sie startet mit der Artikelauswahl und endet mit dem Abschluss des Rüstvorgangs. Die beschriebenen Aspekte tragen außerdem zu einer Aufwandsoptimierung im Zuge der Aufgabenerledigung bei. Anzulernende Maschineneinrichter können bei der Auswahl des ab- und aufzurüstenden Artikels auf bestehende, durch erfahrene Maschineneinrichter bzw. Vorgesetzte angelegte Rüstinstruktionen zurückgreifen.

Die sich im Sichtfeld befindlichen Hologramme inklusive der darin implementierten Buttons bzw. der vorhandenen Auswahlfunktionen, die zusätzlich durch Hilfefunktionen in Form von Tooltips ergänzt wurden, sind eindeutig ersichtlich und damit selbstbeschreibungsfähig. Der Nutzer kann darüber hinaus mithilfe der Schritt-für-Schritt-Anzeige und durch das „Augmented Machine“-Prinzip als einen „Breadcrumb trail“ jederzeit den aktuellen Prozessstatus und -fortschritt einsehen. Ein „Breadcrumb trail“ stellt eine Art Navigationsverlauf dar, der dem Nutzer anzeigt, an welchem Punkt er sich innerhalb der Software bzw. Applikation befindet.

Aus dem Arbeitsgebiet „Rüstprozess“ - insbesondere in Bezug auf spezifische Maschinen- und Werkzeugelemente des Rotationszugbiegens - verwendete Begriffe, wie „Montageposition“, „Dorn“, „Bolzen“, etc., Bezeichnungen und Konventionen sowie die schrittweise Anleitung zum Rüstprozess einer Produktionsmaschine sind erwartungskonform, da sie eine aufgaben- und nutzungsreihenfolge-bezogene Strukturierung verfolgen. Ferner ist eine eindeutige Interpretierbarkeit der Informationen hinsichtlich einer interkulturellen Verständlichkeit sowie der Verwendung von medialen Inhalten bzw. dynamischen Medien gegeben. Die Montagepositionen der einzelnen Werkzeuge können zudem mithilfe einer über dem Montageraum platzier- 
ten 3D-Kamera überprüft und an den Nutzer zurückgemeldet werden, damit dieser etwaige fehlerhafte Positionen korrigieren kann. Weiterhin leuchten angewählte Widgets auf, sodass eine sofortige und angemessene Rückmeldung auf Nutzerhandlungen generiert wird. Dateneingaben, wie bspw. die Änderung eines Artikelnamens, können mithilfe einer digitalen Tastatur erfolgen, die den lokalen Sprachkonventionen einschließlich sprachspezifischer Buchstaben entspricht. Das System ermöglicht dem Nutzer durch Aufbau und Struktur eine konsistente und routinierte Arbeitsausführung über vergleichbare Rüstvorgänge hinweg.

Der Maschineneinrichter ist in der Lage, das System flexibel und individuell zu steuern. Neben einem beliebigen schrittweisen Wechsel der Rüstschritte können Fenstergrößen skaliert bzw. angepasst sowie Hologrammpositionen und -ansichten verändert werden. Es obliegt dem Nutzer, ob und wie bzw. in welchem Ausmaß er die zur Verfügung stehenden Instruktionen nutzt. Beispielsweise kann der Maschineneinrichter das Video beliebig oft ansehen. Ein weiterer Aspekt ist, dass zugunsten der InteraktionsGeschwindigkeit Suchfunktionen mit integrierter Auto-Vervollständigungsfunktion implementiert werden, z. B. bei der Auswahl des gewünschten Artikels. Außerdem können u.a. Hintergrund-, Fokussierungs- und Schriftfarbe gemäß der ArbStättV (2004) individuell konfiguriert werden.
Das CPS-Tool unterstützt den Nutzer bei der Entdeckung seiner Fähigkeiten sowie hinsichtlich eines Lerneffekts. Zunächst liegt der Schwerpunkt der Aufmerksamkeit auf den im oberen Sichtfeld zentriert dargestellten Informationen. In diesem Zusammenhang kann der Nutzer Informationen wiederholt abrufen, wie z.B. das Video des jeweiligen Rüstschritts. Bei jeder Eingabe des Nutzers gibt das System einen Audioton wieder, sodass eine erfolgreiche Interaktion bestätigt wird.

Durch eine sensorische Prüfung der Positionen der montierten Werkzeuge hat der Maschineneinrichter weiterhin die Möglichkeit, den Prozess zu explorieren und letztlich zu optimieren. Die kontextspezifische, detaillierte und problemorientierte Aggregation von realen und virtuellen Daten - zusammen mit sensorischen Eingangswerten zum Rüstprozess - trägt aufgrund einer entsprechend guten Datenstruktur dazu bei, dass eine lernfreundliche Umgebung geschaffen wird. Weiterhin wird hinsichtlich der Rüstanweisungen im Sinne eines Best-Practice-Ansatzes ein iterativer Ansatz verfolgt, der es erlaubt, diese Instruktionen auch mithilfe von Sensortechnologie zu evaluieren und zu modifizieren. Durch sensorbasierte Bewegungsverfolgung oder „Wegtracking“ der HoloLens können im Zuge des Rüstvorgangs entstandene Laufwege aufgezeichnet und in Form eines Videos oder eines Spaghetti-Diagramms aus-

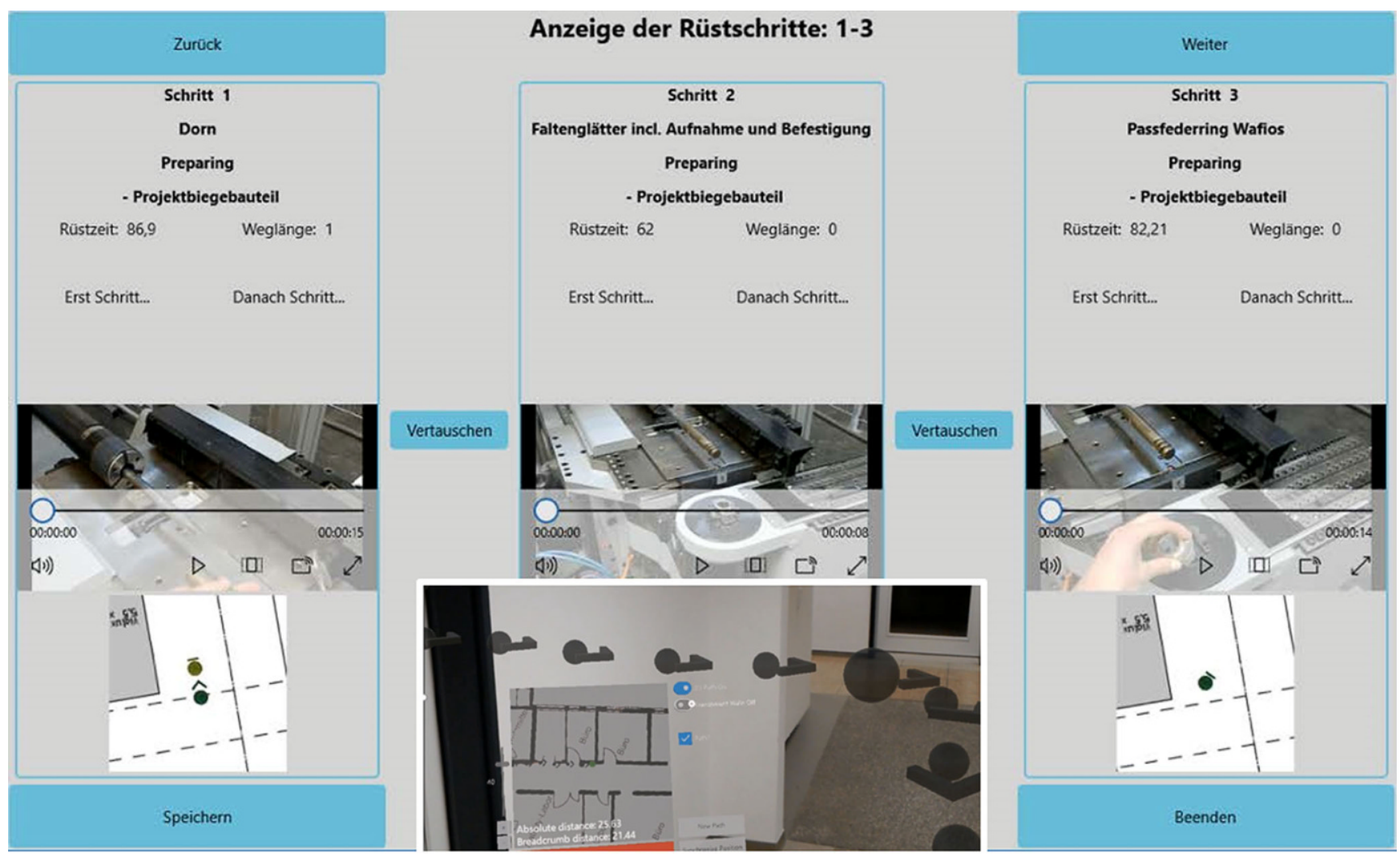

Abb. 8 Exemplarische Darstellung zur Anpassung bzw. Optimierung des Rüstprozesses mithilfe des Tablet-Clients

Fig. 8 Exemplary representation of adapting or optimizing the set-up process via the tablet client 
gegeben werden. Ein implementierter Tablet-Client (vgl. Abb. 8) ermöglicht auf Grundlage dieser Daten eine Optimierung der Rüstschrittreihenfolge gemäß eines ,Single Minute Exchange of Die“ (SMED)-Ansatzes. Dieser zielt durch organisatorische und technische Maßnahmen auf die Minimierung der Rüstzeit in mehreren iterativen Schritten auf bis zur Hälfte der ursprünglich benötigten Zeit ab. Somit kann ein Lernprozess zusätzlich und aktiv unterstützt werden.

Fehler können durch die beschriebenen Unterstützungsmerkmale des „Expert to Go“, u.a. durch die Darstellung aufgabenbezogener Informationen zur Minimierung von Ablenkungen, vermieden werden. Sollten Werkzeuge dennoch fehlerhaft gerüstet worden sein, werden diese durch eine sensorische Überprüfung (der Werkzeugposition) aufgedeckt, um sie anschließend beheben zu können. Eine Tolerierung fehlerhafter Positionen könnte zu einer kostspieligen Zeit- und Ressourcenverschwendung im weiteren Prozessverlauf führen. In Bezug auf die Interaktion mit den Interfaces kann das versehentliche Schließen eines Hologramms durch das Home-Menü rückgängig gemacht werden.

Der Nutzer erhält nach erfolgreicher Durchführung des Rüstprozesses ein Feedback zur Montageposition der einzelnen Werkzeuge. Wenn der Sensor keinen Fehler erkennt, ist der mechanische Rüstvorgang abgeschlossen. Dem Nutzer wird damit eine erfolgreiche Arbeit bescheinigt. Durch die kontextsensitive Bereitstellung der Daten und ihre Ausrichtung auf die Unterstützung von Schlüsselelementen der Entscheidungsfindung wird eine Komplexitätsreduktion erreicht. Dadurch werden keine unverhältnismäßigen Anforderungen an den Nutzer gestellt. Die Neuartigkeit des HMD mitsamt der notwendigen Interaktion und der intuitiven Bedienbarkeit führen weiterhin dazu, dass das interaktive System für die Anwender attraktiv ist. Die Maschineneinrichter werden außerdem stark in das System eingebunden. Während erfahrene Maschineneinrichter Instruktionen erstellen bzw. anlegen, können anzulernende Bediener diese ohne Probleme nutzen. Beide Parteien haben ergänzend dazu die Möglichkeit, bspw. mithilfe des Tablet-Clients, Optimierungen bzw. Anpassungen kollaborativ zu erarbeiten und das System somit laufend zu verbessern. Diese kooperative Zusammenarbeit sowie die jeweils zugeordneten Rollen tragen $\mathrm{zu}$ einer Motivationssteigerung bei.

\section{Diskussion und Ausblick}

Die Bedienerführung moderner Endgeräte, Anlagen und Maschinen nimmt durch den nach wie vor fortschreitenden digitalen Wandel einen großen Stellenwert ein. Neben der Gebrauchstauglichkeit, der Bedienbarkeit und der Verständlichkeit sind auch die Erlernbarkeit sowie eine starke
Verzahnung von Aktion und Reaktion wesentliche Aspekte für eine der menschlichen Informationsverarbeitung zuträglichen Gestaltung moderner Systeme.

AR wird in diesem Zusammenhang zunehmend zur Anleitung und Unterstützung bei Montage- und Rüstvorgängen sowie zur Fehlersuche in industriellen Umgebungen eingesetzt. Das im Zuge des Forschungsprojekts „Cyberrüsten 4.0 “ entwickelte Unterstützungssystem hat gezeigt, dass Nutzer mithilfe der Applikation, mit der sie über ein HMD bzw. die Microsoft HoloLens interagierten, im Vergleich zu herkömmlichen Instruktionsmaterialien, wie papier-basierten Anleitungen, effizientere Arbeitsergebnisse erzielen, u. a. in Form einer minimierten Fehlerrate (Abele und Kluth 2021b). Aufgrund der intuitiven Bedienung sowie der intensiven und konzentrierten Auseinandersetzung mit dem Unterstützungssystem, setzt schnell ein Lerneffekt ein - sowohl hinsichtlich des Rüstprozesses als auch in Bezug auf den Umgang mit der Datenbrille und der Applikation. Die AR-Technologie verhilft in Kombination mit einer wiederholten Ausführung der Arbeitsaufgabe dazu, dass die kognitive Beanspruchung des Nutzers reduziert wird. Gleichzeitig beugt sie potenziell einsetzenden Konzentrationsnachlässigkeiten vor (Lewis and Neider 2016) und unterstützt den Nutzer, prozessrelevantes Wissen zu erlangen bzw. weiterzugeben.

Ohne eine menschzentrierte und kompatible Gestaltung der Informationen hätten die im vorherigen Absatz genannten Erkenntnisse, die von Abele und Kluth (2021a) sowie Abele and Kluth (2021b) im Rahmen von Laborstudien ermittelt wurden, nicht generiert werden können. Für die Praxis nehmen diese Aspekte eine wichtige Rolle ein. Einerseits unterstützt das vorgestellte System unerfahrene bzw. anzulernende Maschineneinrichter zugunsten eines prozesssicheren und fehlerminimalen Rüstvorgangs. AR-basierte Datenbrillen eröffnen zudem auch Menschen mit geistigen und/oder körperlichen Beeinträchtigungen neue berufliche Perspektiven. Sie können u. a. Menschen mit geringer Prozesskenntnis oder hör- bzw. sehbeeinträchtigte Menschen mit visuellen und auditiven Informationen unterstützen. Andererseits kann der aktuelle Forschungsstand (noch) keine signifikante bzw. vollumfängliche Erfolgsgarantie derartiger Tools im betrieblichen Alltag ausweisen. Die Gesetzgebung macht für die Gestaltung neuartiger Visualisierungsarten und speziell für AR-basierte Informationsdarstellungen keine konkreten Vorgaben. Dieser Umstand ist u. a. der rasant fortschreitenden Technisierung geschuldet. Gesetzliche Vorschriften (z. B. ArbStättV 2004), Normen (z. B. DIN EN ISO 9241) oder Leitfäden (z. B. DGUV Information 215410 (2019) oder 215-450 (2021)) dienen für die Gestaltung derartiger Unterstützungssysteme lediglich als übergeordnete und z.T. abstrakte Wegweiser.

Einem eventuellen Effizienzgewinn steht durch den Einsatz eines derartigen CPS-Tools ein möglicher höherer 
Energie- und Ressourcenverbrauch gegenüber. Daher gilt es, die Digitalisierung aktiv zu gestalten, um einerseits ökologische Nachhaltigkeit zu fördern, und andererseits eine nutzerzentrierte bzw. zumutbare und zufriedenstellende Interaktion garantieren zu können. Physische und psychische Beanspruchungen des Nutzers sind darüber hinaus sowohl in Bezug auf die interaktions-ergonomische Gestaltung als auch hinsichtlich produkt-, arbeitssicherheits- und arbeitsumweltrelevanter Aspekte fortwährend zu beachten.

Die vorliegende Ausarbeitung hat am Beispiel einer ARunterstützen Rüstapplikation gezeigt, welche hardware- und software-ergonomischen Kriterien für eine nutzerzentrierte und v.a. interaktions-ergonomische Gestaltung für Informationsdarstellungen zum Tragen kommen. Für einen langfristigen, prozesssicheren, nachhaltigen und aus Sicht der Anwender akzeptierten Einsatz derartiger Systeme sind neben weiteren technologischen Optimierungen und Forschungsbestrebungen bzw. -arbeiten auch gesetzliche und konkrete normative Vorgaben unabdingbar. Dazu sind die Regelungen auf der Grundlage von weiteren Praxisbeispielen bzw. Anwendungsszenarien aus unterschiedlichsten Bereichen zu berücksichtigen, wie z.B. nicht-automatisierte (Fertigungs-)Prozesse, die nach wie vor einen elementaren Bestandteil von KMUs einnehmen.

Danksagung Die vorliegende Ausarbeitung entstammt in Teilen dem Forschungsprojekt „Cyberrüsten 4.0“, das durch die Europäische Union sowie durch den Europäischen Fonds für regionale Entwicklungen des Landes Nordrhein-Westfalen (Nr. EFRE-0800263) finanziert wurde.

\section{Literatur}

Abele ND, Kluth K (2021a) Subjektives Beanspruchungserleben während der Nutzung eines AR-basierten Cyber-Physischen Produktionssystems im Zuge industrieller Rüstvorgänge. In: Proceedings des 67. Frühjahrskongresses der Gesellschaft für Arbeitswissenschaft (GfA). GfA-Press, Dortmund (B.5.1)

Abele ND, Kluth K (2021b) Strain-related evaluation of an AR-based Cyber-physical production system for setting up industrial machines. In: Black N, Neumann P, Noy I (Hrsg) Sector based ergonomics. Proceedings of the 21st Congress of the International Ergonomics Association (IEA 2021b), Bd. 3. Springer, Basel, S 355-362

Abele ND, Hoffmann S, Kuhnhen C, Ludwig T, Schäfer W, Schweitzer M, Wulf V (2016) Supporting the set-up processes by Cyber elements based on the example of tube bending. In: Mayr $\mathrm{CH}$, Pinzger M (Hrsg) INFORMATIK 2016. GI-Edition Lecture Notes in Informatics Proceedings. Köllen Druck+Verlag GmbH, Bonn, S 1627-1636

Apt W, Schubert M, Wischmann S (2018) Digitale Assistenzsysteme Perspektiven und Herausforderungen für den Einsatz in Industrie und Dienstleistungen. Institut für Innovation und Technik (IIT) in der VDI/VDE Innovation + Technik GmbH, Berlin

Balzert H (1987) Gestaltungsziele der Software-Ergonomie. In: Schönpflug W, Wittstock M (Hrsg) Software-Ergonomie '87 Nützen Informationssysteme dem Benutzer? Berichte des German Chapter of the ACM. Vieweg+Teubner Verlag, Wiesbaden, S 477-488
BGHM-FI Nr. 0065 (2019) FAQ-Liste zum Einsatz von Datenbrillen an (gewerblichen) Arbeitsplätzen in Holz- und Metallbranchen. Berufsgenossenschaft Holz und Metall (BGHM), Mainz

Bhattacharya B, Winer EH (2019) Augmented reality via expert demonstration authoring (AREDA). Comput Ind 105:61-79

Birkhan C (2008) Smart Production Systems - Intelligente Konzepte zur Gestaltung von Produktionssystemen. Bosch, Kaiserslautern

Bokranz R, Landau K (1991) Einführung in die Arbeitswissenschaft. Eugen Ulmer, Stuttgart

Bullinger HJ (1994) Ergonomie. Produkt- und Arbeitsplatzgestaltung. Teubner, Stuttgart

Caudell TP, Mizell DW (1992) Augmented reality: an application of heads-up display technology to manual manufacturing processes. In: Proceedings of the Twenty-Fifth Hawaii International Conference on System Sciences (HICSS). IEEE, New York, S 659-669

Chen T, Tsai HR (2017) Ubiquitous manufacturing: current practices, challenges, and opportunities. Robot Comput Integr Manuf 45:126-132

Cometti C, Païzis C, Casteleira A, Pons G, Babault N (2018) Effects of mixed reality head-mounted glasses during 90 minutes of mental and manual tasks on cognitive and physiological functions. PeerJ 6(4): 55847

De Carvalho AFP, Hoffmann S, Abele ND, Schweitzer M, Tolmie P, Randall D, Wulf V (2018) Of embodied action and sensors. Knowledge and expertise sharing in industrial set-up. Comput Coop Work (CSCW) 27(3-6):875-916

DGUV (2019) Bildschirm- und Büroarbeitsplätze. DGUV Information 215-410. Deutsche Gesetzliche Unfallversicherung, Berlin

DGUV (2021) Softwareergonomie. DGUV Information 215-450. Deutsche Gesetzliche Unfallversicherung (DGUV), Berlin

DIN EN 894 (2008) Sicherheit von Maschinen - Ergonomische Anforderungen an die Gestaltung von Anzeigen und Stellteilen - Teil 2: Anzeigen. Beuth, Berlin/Köln/Frankfurt a.M.

DIN EN ISO 9241 (2008) Ergonomie der Mensch-System-Interaktion - Teil 307: Analyse- und Konformitätsverfahren für elektronische Anzeigen. Beuth, Berlin/Köln/Frankfurt a.M.

DIN EN ISO 9241 (2011) Ergonomie der Mensch-System-Interaktion - Teil 303: Anforderungen an elektronische optische Anzeigen. Beuth, Berlin/Köln/Frankfurt a.M.

DIN EN ISO 9241 (2017) Ergonomie der Mensch-System-Interaktion - Teil 112: Grundsätze der Informationsdarstellung. Beuth, Berlin/Köln/Frankfurt a.M.

DIN EN ISO 9241 (2017) Ergonomie der Mensch-System-Interaktion - Teil 125: Empfehlungen zur visuellen Informationsdarstellung. Beuth, Berlin/Köln/Frankfurt a.M.

DIN EN ISO 9241 (2020) Ergonomie der Mensch-System-Interaktion - Teil 110: Interaktionsprinzipien. Beuth, Berlin/Köln/Frankfurt a.M.

Engel B, Mathes C (2013) Erweiterung der Prozessfähigkeit des Rotationszugbiegens durch ein alternatives Faltenglätterkonzept. Europäische Forschungsgesellschaft für Blechverarbeitung e.V. (EFB), Hannover

Gallagher S (2010) Merleau-Ponty's phenomenology of perception. Topoi Int Rev Philos 29(2):183-185

Gauglitz S, Nuernberger B, Turk M, Höllerer T (2014a) In Touch with the Remote World: Remote Collaboration with Augmented Reality Drawings and Virtual Navigation. In: Proceedings of the 20th ACM Symposium on Virtual Reality Software and Technology (VRST). ACM, New York, S 197-205

Gauglitz S, Nuernberger B, Turk M, Höllerer T (2014b) World-stabilized annotations and virtual scene navigation for remote collaboration. In: Proceedings of the 27th annual ACM Symposium on User Interface Software and Technology (UIST. ACM, New York, S 449-459

Hacker W (1987) Software-Ergonomie; Gestalten Rechnergestützter Geistiger Arbeit? In: Schönpflug W, Wittstock M (Hrsg) Software-Ergonomie '87 Nützen Informationssysteme dem Benut- 
zer? Berichte des German Chapter of the ACM. Vieweg+Teubner, Wiesbaden, S 31-54

Heeg FJ (1988) Empirische Software-Ergonomie. Zur Gestaltung benutzergerechter Mensch-Computer-Dialoge. Springer, Berlin, Heidelberg

Herterich M, Uebernickel F, Brenner W (2015) The Impact of CyberPhysical Systems on Industrial Services in Manufacturing. Procedia CIRP 30:323-328

Hoffmann S, De Carvalho AFP, Abele ND, Schweitzer M, Tolmie P, Wulf V (2019) Cyber-Physical Systems for Knowledge and Expertise Sharing in Manufacturing Contexts: Towards a Model Enabling Design. Comput Coop Work (CSCW) 28:469-509

Janlert LE, Stolterman E (2010) Complex Interaction. ACM Trans Comput Interact 17:1-32

Janssen S, Möller K (2011) Erfolgreiche Steuerung von Innovationsprozessen und -projekten - Ergebnisse einer empirischen Studie. Control Manag 55(2):97-104

Jensen MB, Johnson B, Lorenz E, Lundvall BÅ (2007) Forms of knowledge and modes of innovation. Res Policy 36:680-693

Kagermann H, Wahlster W, Helbig J (2013) Recommendations for Implementing the Strategic Initiative INDUSTRIE 4.0: Final Report of the Industrie 4.0 Working Group. National Academy of Science and Engineering, Frankfurt a.M.

Klopfer E, Perry J, Squire K, Jan M (2005) Collaborative learning through augmented reality role playing. In: Proceedings of the 2005 Conference on computer support for collaborative learning (CSCL): learning 2005: the next 10 years! International Society of the Learning Sciences, , S 311-315

Kober M, Scheve J, Dahm A (2014) Rüstoptimierung - Das Ziel sind kurze Durchlaufzeiten. Betriebsprax Arbeitsforsch 222:10-15

Lee S, Akin Ö (2011) Augmented reality-based computational fieldwork support for equipment operations and maintenance. Autom Constr 20(4):338-352

Lee J, Bagheri B, Kao HA (2015) A cyber-physical systems architecture for industry 4.0-based manufacturing systems. Manuf Lett $3: 18-23$

Lewis J, Neider M (2016) Through the Google glass: the impact of heads-up-display on visual attention. Cogn Res Princ Implic 1(13): $1-13$

Ludwig T, Kotthaus C, Stein M, Durt H, Kurz C, Wenz J, Doublet T, Becker M, Pipek V, Wulf V (2016) Arbeiten im Mittelstand 4.0 KMU im Spannungsfeld des digitalen Wandels. HMD 53(1):1-16

Matthew CT, Sternberg RJ (2009) Developing experience-based (tacit) knowledge through reflection. Learn Individ Differ 19(4):530-540

Monostori L (2014) Cyber-physical production systems: roots, expectations and R\&D challenges. Procedia CIRP 17:9-13

N.N. (2019) HoloLens-Hardware (1. Generation). https://docs.microsoft. com/de-de/hololens/hololens1-hardware. Zugegriffen: 16. Sept. 2019
Navas BJ (2015) Software-Ergonomie. Grundlagen der optimalen Ausgestaltung von Computersystemen für die Benutzer. GRIN, München

Nonaka I, Toyama R, Konno N (2000) SECI, Ba and leadership: a unified model of dynamic knowledge creation. Long Range Plann 33(1):5-34

Ong SK, Yuan ML, Nee AYC (2008) Augmented reality applications in manufacturing: a survey. Int J Prod Res 46(10):2707-2742

Otto J, Henning S, Niggemann O (2014) Why cyber-physical production systems need a descriptive engineering approach — a case study in plug \& produce. Procedia Technol 15:295-302

Paelke V, Röcker C (2015) User interfaces for cyber-physical systems: challenges and possible approaches. Springer, Cham

Park M, Schmidt L, Schlick C, Luczak H (2007) Design and evaluation of an augmented reality welding helmet. Hum Factors Man 17(4):317-330

Pollalis C, Fahnbulleh W, Tynes J, Shaer O (2017) Holomuse: enhancing engagement with archaeological artifacts through gesturebased interaction with holograms. In: Proceedings of the tenth international conference on tangible, embedded, and embodied interaction (TEI). ACM, New York, S 565-570

Schmauder M, Spanner-Ulmer B (2014) Ergonomie. Grundlagen zur Interaktion von Mensch, Technik und Organisation. Hanser, München

Shi J, Wan J, Yan H, Suo H (2011) A survey of Cyber-physical systems. In: Proceedings of the wireless communications and signal processing conference (WCSP). IEEE, New York

Stanney K (2002) Handbook of Virtual Environments. Design, Implementation, and Applications. Lawrence Erlbaum, Mahwah

Strasser H (1993) Ergonomie - Arbeitsplatz. Kap. 2.4.5. Kompatibilität. In: Hettinger T, Wobbe G (Hrsg) Kompendium der Arbeitswissenschaft. Kiel-Verlag, Ludwigshafen, S 228-242

Thramboulidis K, Christoulakis F (2016) UML4IoT-A UML-based approach to exploit IoT in Cyber-physical manufacturing systems. Comput Ind 82:259-272

VDI 3430 (2014) Rotationszugbiegen von Profilen, Beuth, Berlin/ Köln/Frankfurt a.M.

Verordnung über Arbeitsstätten (ArbStättV) vom 12. August 2004, zuletzt geändert durch Artikel 4 des Gesetzes vom 22. Dezember 2020, BGB1. I, S 3334

Womack JP, Jones DT, Roos D (1990) The machine that changed the world: the story of lean production. Westview Press, Boulder

Wulf V, Müller C, Pipek V, Randall D, Rohde M, Stevens G (2015) Practice-based computing: empirically grounded conceptualizations derived from design case studies. In: Wulf V, Schmidt K, Randall D (Hrsg) Designing socially embedded technologies in the real-world. Springer, London, S 111-150

Zidek K, Pitel J, Adamek M, Lazorik P, Hosovsky A (2020) Digital twin of experimental smart manufacturing assembly system for industry 4.0 concept. Sustainability 12(9):3658 